\title{
Catastrophic disruptions as the origin of bilobate comets
}

\author{
Stephen R. Schwartz ${ }^{1,2 \star}{ }^{1}$, Patrick Michel', Martin Jutzi ${ }^{3}$, Simone Marchi ${ }^{4}$, Yun Zhang ${ }^{5,6}$ \\ and Derek C. Richardson ${ }^{6}{ }^{6}$
}

\begin{abstract}
Several comets observed at close range have bilobate shapes ${ }^{1}$, including comet 67P/Churyumov-Gerasimenko (67P/C-G), which was imaged by the European Space Agency's Rosetta mission $^{2,3}$. Bilobate comets are thought to be primordial because they are rich in supervolatiles (for example, $\mathbf{N}_{2}$ and CO) and have a low bulk density, which implies that their formation requires a very low-speed accretion of two bodies. However, slow accretion does not only occur during the primordial phase of the Solar System; it can also occur at later epochs as part of the reaccumulation process resulting from the collisional disruption of a larger body ${ }^{4}$, so this cannot directly constrain the age of bilobate comets. Here, we show by numerical simulation that $67 \mathrm{P} / \mathrm{C}-\mathrm{G}$ and other elongated or bilobate comets can be formed in the wake of catastrophic collisional disruptions of larger bodies while maintaining their volatiles and low density throughout the process. Since this process can occur at any epoch of our Solar System's history, from early on through to the present day ${ }^{5}$, there is no need for these objects to be formed primordially. These findings indicate that observed prominent geological features, such as pits and stratified surface layers ${ }^{4,5}$, may not be primordial.
\end{abstract}

To investigate the effects of catastrophically disruptive impacts on small outer Solar System bodies, including heating, compaction and the shapes of reaccumulated aggregates, we combined the results of a smoothed-particle hydrodynamics (SPH) code that simulates the fragmentation phase of the disruption and an $\mathrm{N}$-body code to simulate the ensuing phase of gravitational reaccumulation. We performed a series of simulations in which we varied the projectile-to-target mass ratio and the impact speed. Table 1 presents the different impact conditions used in our investigation. Although constrained by computational expense, the initial conditions that we chose cover a range of impact speeds that are encountered either in the early Solar System or at later stages. In all cases, they occur at an impact energy that is above the catastrophic disruption threshold so that our results focus on the process of catastrophic disruption.

For the simulations of the fragmentation phase, the objects were initially spherical and made of ice, with an initial density of $0.5 \mathrm{~g} \mathrm{~cm}^{-3}$. Porosity was modelled using a P-alpha model $^{6}$ with a simple quadratic relation using crush curve parameters $P_{\mathrm{e}}=10^{4} \mathrm{~Pa}$ and $P_{\mathrm{s}}=10^{6} \mathrm{~Pa}$, where pressures leave the purely elastic phase above $P_{\mathrm{e}}$ leading to irreversible compaction, and where full compaction is reached at pressures larger than $P_{s}$. We used a tensile fracture model $^{7}$ with parameters that lead to an average tensile strength $Y_{\mathrm{T}}$ of $\sim 50 \mathrm{kPa}$ (this is larger than the bulk strength inferred in other studies ${ }^{8}$ from the tensile strength between the two components of 67P). We note that the collisions considered here can lead to a complete fragmentation of the targets. As shown in previous studies of the disruption regime ${ }^{9}$, the outcomes of such events are not sensitive to the tensile strength. To model fractured, granular material, a pressure-dependent shear strength (friction) was included using a standard Drucker-Prager yield criterion. We used the Tillotson equation of state with parameters for water-ices. This equation of state is adequate for modelling the collisions considered here because the most important response is the compressibility of the material, which is given by the crush curve of the P-alpha model.

To compute an estimate of the temperature increase of the material, $\Delta T$, as a result of the collision, we converted the increase in the specific internal energy, $\Delta u$, to a temperature increase using the equation $\Delta T=\Delta u / c_{\mathrm{p}}$, where $c_{\mathrm{p}}$ is the constant heat capacity, equal to $100 \mathrm{~J} \mathrm{~kg}^{-1} \mathrm{~K}^{-1}$ ( ref. $^{9}$ ) - a mass-weighted average of the heat capacity of ice and silicates. We stress that this approximation only yields reasonable values provided $\Delta T$ is small ( $\sim 100 \mathrm{~K}$ or less). For higher temperatures, $\Delta T$ is overestimated due to the temperature dependence of $c_{\mathrm{p}}$.

The fragmentation process was integrated for $300 \mathrm{~s}$ of simulated time, well beyond the point at which the fracture ceased. From here, we defined a constituent particle distribution that included the body's fracture state, velocity distribution and density distribution. We then ported this result into our $\mathrm{N}$-body code PKDGRAV $^{10,11}$ as an ensemble of discrete soft-sphere particles ${ }^{12}$ using a procedure detailed in previous work ${ }^{13}$, which includes avoiding overlapping particles.

The material friction resistance was controlled by several friction parameters given in our $N$-body model ${ }^{12,14}$. To show that the validity of our results can be extrapolated to the wide range of friction parameters that may describe these bodies, we intentionally looked at the low end of interparticle friction and used non-cohesive granular material with a friction angle of $18^{\circ}$. Real comets therefore hold their shapes better than the aggregates in our study ${ }^{15}$, showing even greater deviation from sphericity and irregular features such as those on 67P.

Our simulations of catastrophic disruptions showed that much of the material from the progenitor bodies reaccumulates after disruption-not necessarily into a single body, but into many smaller bodies. Next, we analysed the shapes and sizes of the reaccumulated aggregates for each simulation. Figure 1 shows that even with relatively low friction and no cohesion between components, dispersed fragments came together to form non-spherical aggregates. Fitting

'Université Côte d'Azur, Observatoire de la Côte d'Azur, Centre National de la Recherche Scientifique, Laboratoire Lagrange, Nice, France. ${ }^{2}$ Lunar and Planetary Laboratory, University of Arizona, Tucson, AZ, USA. ${ }^{3}$ Physics Institute, University of Bern, National Centre of Competence in Research PlanetS, Bern, Switzerland. ${ }^{4}$ Southwest Research Institute, Boulder, CO, USA. ${ }^{5}$ School of Aerospace Engineering, Tsinghua University, Beijing, China. ${ }^{6}$ Department of Astronomy, University of Maryland, College Park, MD, USA. *e-mail: srs@oca.eu 
Table 1 | Simulations of impact disruptions of comet-like bodies

\begin{tabular}{lllll}
$\begin{array}{l}\text { Target-to- } \\
\text { projectile } \\
\text { mass ratio }\end{array}$ & $\begin{array}{l}\text { Impact } \\
\text { speed } \\
\left(\mathbf{m ~ s}^{-1}\right)\end{array}$ & $\begin{array}{l}\text { Impact angle } \\
\text { (degrees) }\end{array}$ & $\begin{array}{l}\text { Total } \\
\text { particles }\end{array}$ & $\begin{array}{l}\text { Total mass } \\
(\mathbf{k g})\end{array}$ \\
\hline $1 / 1$ & 20 & 45 & 452,544 & $1.131 \times 10^{14}$ \\
$2 / 1$ & 50 & 45 & 452,482 & $1.129 \times 10^{14}$ \\
$8 / 1$ & 150 & 45 & 451,951 & $1.128 \times 10^{14}$ \\
$27 / 1$ & 300 & 45 & 453,092 & $1.127 \times 10^{14}$ \\
$27 / 1$ & 1,000 & 52 & 453,092 & $1.127 \times 10^{14}$ \\
\hline
\end{tabular}

Comet $67 \mathrm{P}$ has a mass of $\sim 10^{13} \mathrm{~kg}^{2}$-about $9 \%$ of the total progenitor mass in these simulations. Mass ratios and impact angles were chosen to obtain roughly the same degree of disruption in each case.

the aggregate shapes as prolate ellipsoids, the principal axes were on average about $50 \%$ greater than their minor axes, but with significant spread. This is in line with what we see in the comet population. Although much of the material contributed to the largest, often elongated remnant, unbound or loosely bound material was also produced, leading to the formation of a multitude of smaller gravitational aggregates (see Supplementary Videos 1-3). We found the specific end-state to be chaotic as it was extremely dependent on initial impact conditions. The extent to which these elongated bodies that form the next generation of comets are made up of distinct 'lobes' depends on (1) whether they originate from two already formed distinct aggregates that merge together and (2) the frictional

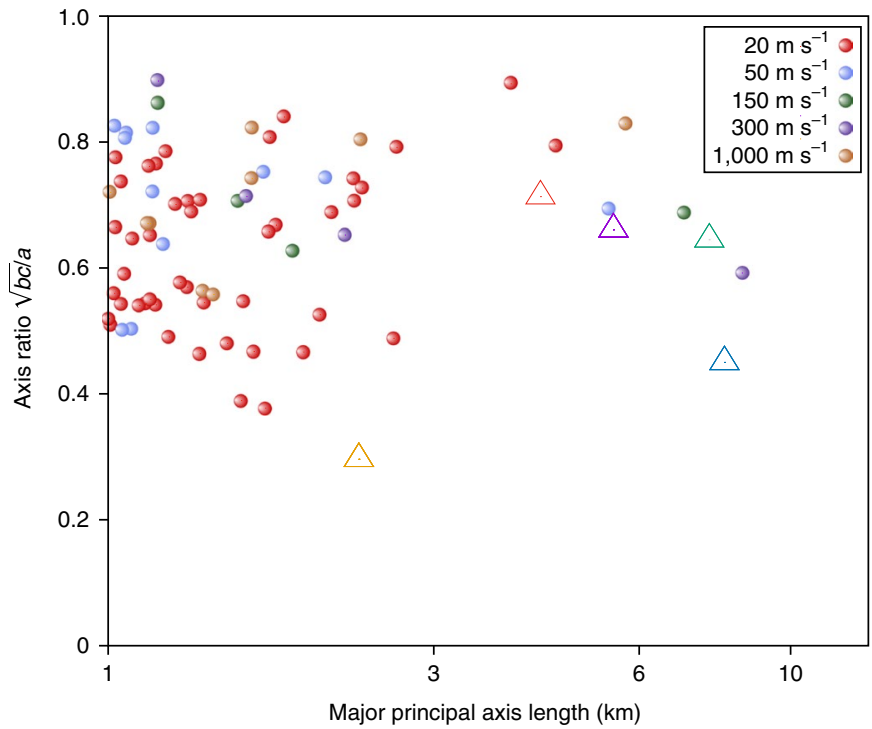

Fig. 1 | Ratio of minor to major principal axes for each reaccumulated body with major axes over $1 \mathrm{~km}$, when fit to a prolate spheroidal shape. These bodies are typically elongated and irregularly shaped, commensurate with what has thus far been observed of the cometary population ${ }^{29-31}$. They are also often composed of fragments that originated from regions near to each other inside the progenitors. Aggregates show a distribution of shapes and sizes, with width-to-length ratios ranging from 0.4-0.9. The elongated shapes are the result of smaller, transitory reaccumulated aggregates that combine at late stages of the reaccumulation process. Friction parameters determine how well these transitory aggregates retain their shapes after combining, and the extent of their bilobate configurations. Shapes and sizes of five comets are also plotted for comparison (open triangles); from left to right, these are: Hartley-2 (ref. ${ }^{31}$; orange), 67P (ref. ${ }^{2}$; red), Wild 2 (ref. ${ }^{32}$; purple), Tempel 1 (ref. ${ }^{30}$; green) and Borelly ${ }^{33,34}$ (blue). properties of the material, namely on the capability of preserving the shapes of the two components while they merge. Therefore, the friction parameters determine how bilobate the final aggregate is. For instance, an angle-of-friction of $29^{\circ}$ was needed to form the bilobate shape of Supplementary Video 2. As can be seen from Supplementary Video 1, a lower angle-of-friction of $18^{\circ}$ resulted in a more ellipsoidal shape. Supplementary Video 4 shows another example of bilobate body formation that involves the merging of two equally massive aggregates at a late stage in the reaccumulation process to form a final body with mass close to that of 67P. Its shape depends on the conditions of the collision and the friction parameters of the material.

Although we simulated impacts that dispersed the progenitors into many fragments, we found that this did not produce a great deal of heating throughout the body (Supplementary Fig. 1), allowing volatile species to survive the process. Of even more significance, the relatively small fraction of material that was heated substantially was also ejected at higher speeds and did not reaccumulate (Fig. 2; this also agrees with recent work ${ }^{10}$ involving less energetic collisions). Figure 2 distinguishes material contained in reaccumulated aggregates based on heating, showing the fraction of material inside aggregates that were heated by a given amount. We found that the amount of heating that a material undergoes correlates tightly with its velocity dispersion, which explains why material that suffers significant heating does not end up in reaccumulated aggregates, small or large. In fact, Fig. 2 also shows that because of this correlation of heating and dispersion, smaller aggregates ('smaller' relative to the resolution in these simulations) contained the least heated material-even less than the material that ended up in the lessgravitationally-weak larger remnants. Therefore, after large-scale catastrophic disruptions, significantly heated material will not be found in the next generation of comets. This suggests that the volatile compounds found inside cometary nuclei can subsist through multiple collisions, including highly energetic catastrophic events, throughout the age of the Solar System.

Similarly, in regard to pore preservation after catastrophic disruption, the regions where material is compacted are typically regions of gravitationally unbound material with a higher initial relative speed that do not easily reaccrete into aggregates. We found that the largest aggregates resulting from the catastrophic disruptions

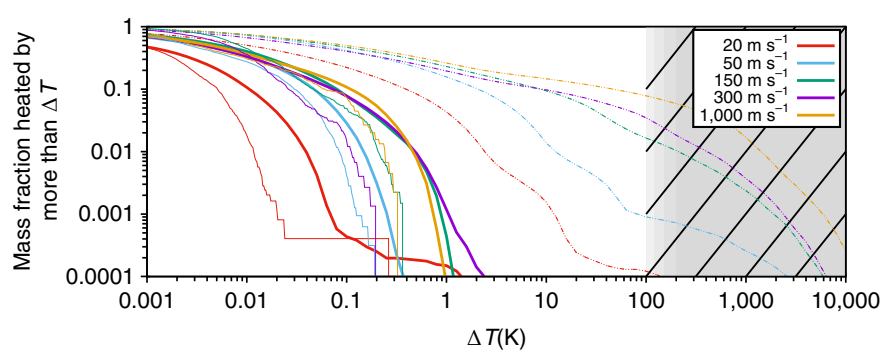

Fig. 2 | Temperature increases resulting from catastrophic collisions at a range of impact speeds. Coloured lines indicate the fraction of material whose temperature rose above the given amount. Dashed lines represent all material from the progenitor bodies, while solid lines represent material that ended up in reaccumulated aggregates. Thick solid lines indicate the temperature increases for material in the larger reaccumulated aggregates shown in Fig. 1. Thin solid lines represent material in small aggregates (the fifty-first to one-hundredth most massive aggregates). When calculating temperature increases, we use a heat capacity relevant for low-temperature material-our focus - thus the data on large temperature increases are not reliable (struck-through region above $\sim \Delta T=100 \mathrm{~K}$ ). See Supplementary Fig. 3 for temperature increases for material inside the largest remnant, including the results from a $3 \mathrm{~km} \mathrm{~s}^{-1}$ catastrophic disruption scenario. 
investigated here experienced no significant compaction (Fig. 3). Moreover, the creation of macroporosity by gentle reaccumulation of ejected material can lead to an additional increase in the overall porosity by $20-30 \%{ }^{16}$. During the collision phase, dilation due to shear failure may further increase porosity ${ }^{17}$.

Catastrophic and marginally catastrophic collisions produce material straddling the limits of their mutual gravity, leading to low-speed mergers and a great variety of final shapes and sizes; this can account for what we observe in today's cometary landscape. Clusters of fragments that initially separate at low speeds relative to each other coalesce during the gravitational phase into the next generation of comets. The relative speeds of these fragments as they reaccumulate are thus also necessarily low (typically under $1 \mathrm{~m} \mathrm{~s}^{-1}$ for progenitors twice the size of $67 \mathrm{P}^{18,19}$ ). This allows transient aggregates to keep a memory of their shapes and porosities as they grow during the reaccumulation phase ${ }^{18}$. Our simulations show that small fragments gently reaccrete to form many transitory aggregates in the hours and days after a disruption. The eventual shape is often the result of two or more large transitory aggregates coming together and pressing into each other.

Our results offer a clear solution to the problem of the survivability of observed comets ${ }^{5,9,20}$ since collisions with energies sufficient to produce such disruption events could have taken place relatively late in the life of the Solar System. We note that the bilobate or elongated shapes resulting from the disruption of a large body might further evolve in subsequent smaller-scale collisions ${ }^{16}$ and/or fission-merging cycles ${ }^{8}$. Although a recent analysis of crater distributions on Pluto and Charon ${ }^{21}$ suggests a depletion of small $(<1 \mathrm{~km})$ trans-Neptunian objects, this would reduce the number of reshaping collisions but have little effect on the number of catastrophic collisions. Thus far, the specific features contained in the images of $67 \mathrm{P}$ and resolved by the Rosetta team ${ }^{22}$ are also commensurate with the catastrophic disruption scenario. Catastrophic collisions could potentially help to explain puzzling observations, such as layering and large-scale fracturing, which otherwise remain a mystery ${ }^{5}$. Widespread fracturing observed in the Hathor region of $67 \mathrm{P}$ could

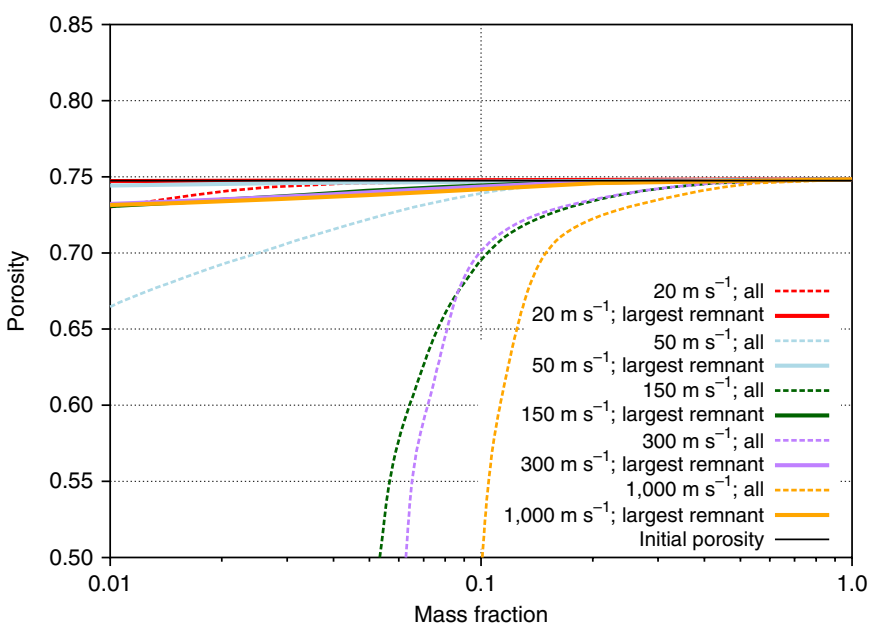

Fig. 3 | Post-impact porosity distributions showing the amounts of mass at a given porosity or below for the catastrophic disruption simulations used in this study. Dashed lines represent material from the entire simulation, while solid lines represent material comprising only the largest remnant. Following previous studies concerning the distribution of compacted material after an impact ${ }^{16}$, a reference density for fully compacted material of $1,980 \mathrm{~kg} \mathrm{~m}^{-3}$ was used, which yielded an initial porosity of $75 \%$. Supplementary Fig. 4 includes the results for a $3 \mathrm{~km} \mathrm{~s}^{-1}$ disruption. be the result of coherent cracking upon impact, provided a competent piece of the parent body can survive the event. Layering on the comet ${ }^{3}$ could occur at the end of the reaccumulation process. During this late stage, in the days and weeks following the disruption, and after the body has achieved its overall shape, small aggregates in the vicinity typically continue to reaccumulate onto it in an analogous manner to catastrophic disruption and reaccumulation studies of the inner Solar System ${ }^{4}$. This continuous process contributes a layering of material onto the surface. In reality, this lateaccreting material may be flattened upon impact with the surface ${ }^{18}$, leading to a layered structure. Moreover, the accumulation of large blocks at this stage would result in the presence of significant cavities, which could provide nucleation seeds for the large pits ${ }^{23}$. Thus, in our scenario, the majority of geological features originate naturally from the reaccumulation phase in the wake of a catastrophic disruption event. Current observations should have a limited bearing on the nature of the primordial assemblages. In contrast, since our simulations show that the material itself does not experience significant heating or compaction, its primordial composition remains preserved, in agreement with the classification of comets as primitive bodies $^{24}$. Although today's catalogue of well-characterized cometary shapes is mostly limited to those we have visited by spacecraft, what we have seen paints a picture that matches a formation scenario involving catastrophic disruption.

\section{Methods}

We combined the results of an SPH code that simulates the fragmentation phase of the disruption process and an $N$-body code (PKDGRAV) to simulate the ensuing phase of gravitational reaccumulation. We performed a series of simulations in which we varied the projectile-to-target mass ratio and impact speed (see main text). The rationale for this hybrid approach is that timescales of fragmentation are very short (typically a few seconds for a kilometre-sized target) compared with the gravitational phase (several days or longer). The computational effort necessary to resolve the impact-induced fragmentation phase in SPH cannot be carried out to simulate the days-long gravitational reaccumulation phase. Also, after fragmentation, the physical regime becomes one dominated by gravity and granular interactions, precisely suited for PKDGRAV. This is a similar approach to one taken for the case of asteroid disruption and reaccumulation ${ }^{25}$, but with meaningful differences (see below) in addition to significant updates to both codes.

The gravitational reaccumulation of material after catastrophic disruption to form asteroid families ${ }^{25}$ was computed either using a merging procedure that combined two spheres into a single sphere (with a mass of the two colliding spheres) or using a model of rigid aggregates ${ }^{26}$ that did not truly solve for the contact forces between the reaccumulated particles ${ }^{4}$. We kept track of temperature increases down to particle-scale resolution (around tens of metres; see Supplementary Fig. 1) and used the soft-sphere discrete element method to compute the shapes of resultant aggregates during and after gravitational reaccumulation (Supplementary Fig. 2), as described below.

The fragmentation phase was computed with an improved version of a previous SPH hydrocode ${ }^{7}$, which included a fracture, a friction and a porosity model ${ }^{6,27}$. The ensuing gravitational phase, during which fragments interacted under their mutual gravity and potentially reaccumulated, was computed with the parallel $\mathrm{N}$-body gravity tree-code PKDGRAV ${ }^{10,11}$. Since our objective was to determine whether bilobate shapes are produced during disruptive events, we needed to explicitly treat the contacts between fragments that reaccumulated so that we could solve for their final shapes in a more realistic way. Thus, we used a version of PKDGRAV that included the soft-sphere discrete element method ${ }^{12,28}$, while keeping track of all temperature and porosity alterations.

Colliding particles in the soft-sphere discrete element method are permitted to overlap by a small amount (typically $<1 \%$ of their radii), which can be considered a proxy for the deformation real particles undergo upon collision. The overlapping of particles induces a repulsive force between them that increases with the extent of the overlap. Coefficients of restitution and other material properties (for example, surface roughness and deviation from sphericity) define the magnitudes of the dissipative and frictional forces particles are subject to when in contact (further details of the collisional methodology $y^{12}$ and explanations of the tree-code methodology and neighbour-finder routines ${ }^{10,11}$ are available). In place of the earlier implementation of the rolling friction routine ${ }^{12}$, we used a more rigorous routine $\mathrm{e}^{14}$, but with the interparticle contact radius in place of the reduced radius for force calculations. Using the radius of contact between particles to compute rolling friction forces rather than the reduced radius causes rolling friction to increase more severely with the extent of overlap and requires a greater value of the parameter $\beta$, which quantifies angularity, to achieve the same rolling friction. 
The limits of our current knowledge about the mechanical properties of cometary material mean that the material parameter values are not well defined. Throughout our simulations, we used intermediate values for the normal and tangential restitution coefficients: 0.5 and approximately 0.8 , respectively (the coefficient of tangential restitution is not explicitly solved in our soft-sphere discrete element method implementation; see additional discussion in ref. ${ }^{12}$ ). For the stiffness parameters $k_{\mathrm{n}}$ and $k_{\mathrm{t}}$, which define the grains' resistance to normal- and tangential-axis compression, respectively, we used values of $10^{8} \mathrm{~kg} \mathrm{~s}^{-3}$ and $2.86 \times 10^{7} \mathrm{~kg} \mathrm{~s}^{-3}$. To control the number of variables, we kept the twisting friction parameter $\mu_{\mathrm{t}}=0$. Except when otherwise indicated, we set the Coulomb friction parameter $\mu_{\mathrm{s}}=1.0$, the rolling friction parameters $\mu_{\mathrm{R}}=0.5$ and $\beta=5.0$, and the timestep $\Delta t=0.5 \mathrm{~s}$. Many of these values were arrived at through physical constraints (overlaps between colliding particles must be constrained by the choice of $k_{\mathrm{n}}$ and $k_{\mathrm{t}}$, which in turn determines an upper limit to the timestep), experimentation, and accumulated experience from previous studies-they represent a set of nominal material properties, but future studies could explore a larger parameter space. Also for the sake of limiting the parameter space, and to highlight the generality of our results, we did not include the effects of cohesion between grains or a large size distribution of particles in this study. Both of these are important effects for maintaining the shapes of the fragments after low-speed accumulation. To link the material properties of our simulated aggregates to granular materials, we carried out stress analyses on the aggregates to compute the friction angle for each case $^{14}$ (Supplementary Fig. 2)

Data availability. The data that support the plots within this paper and other findings of this study are available from the corresponding author upon reasonable request.

Received: 6 March 2017; Accepted: 23 January 2018; Published online: 05 March 2018

\section{References}

1. Sunshine, J. M., Thomas, N., El-Maarry, M. R. \& Farnham, T. L. Evidence for geologic processes on comets. J. Geophys. Res. Planets 121, 2194-2210 (2016).

2. Sierks, H. et al. On the nucleus structure and activity of comet 67P/ Churyumov-Gerasimenko. Science 347, aaa1044 (2015).

3. Massironi, M. et al. Two independent and primitive envelopes of the bilobate nucleus of comet 67P. Nature 526, 402-405 (2015)

4. Michel, P. \& Richardson, D. C. Collision and gravitational reaccumulation: possible formation mechanism of the asteroid Itokawa. Astron. Astrophys. 554, L1 (2013).

5. Rickman, H. et al. Comet 67P/Churyumov-Gerasimenko: constraints on its origin from OSIRIS observations. Astron. Astrophys. 583, A44 (2015).

6. Jutzi, M., Benz, W. \& Michel, P. Numerical simulations of impacts involving porous bodies. I. Implementing sub-resolution porosity in a 3D SPH hydrocode. Icarus 198, 242-255 (2008).

7. Benz, W. \& Asphaug, E. Impact simulations with fracture. I. Method and tests. Icarus 107, 98-116 (1994).

8. Hirabayashi, M. et al. Fission and reconfiguration of bilobate comets as revealed by 67P/Churyumov-Gerasimenko. Nature 534, 352-355 (2016).

9. Jutzi, M., Benz, W., Toliou, A., Morbidelli, A. \& Brasser, R. How primordial is the structure of comet $67 \mathrm{P} / \mathrm{C}-\mathrm{G}$ ? Combined collisional and dynamical models suggest a late formation. Astron. Astrophys. 597, A61 (2017).

10. Stadel, J. G. Cosmological N-body Simulations and Their Analysis. PhD thesis, Univ. Washington (2001).

11. Richardson, D. C., Quinn, T., Stadel, J. \& Lake, G. Direct large-scale N-body simulations of planetesimal dynamics. Icarus 143, 45-59 (2000).

12. Schwartz, S. R., Richardson, D. C. \& Michel, P. An implementation of the soft-sphere discrete element method in a high-performance parallel gravity tree-code. Granul. Matter 14, 363-380 (2012).

13. Schwartz, S. R., Yu, Y., Michel, P. \& Jutzi, M. Small-body deflection techniques using spacecraft: techniques in simulating the fate of ejecta. Adv. Space Res. 57, 1832-1846 (2016)

14. Zhang, Y. et al. Creep stability of the proposed AIDA mission target 65803 Didymos: I. Discrete cohesionless granular physics model. Icarus 294, 98-123 (2017).

15. Groussin, O. et al. Gravitational slopes, geomorphology, and material strengths of the nucleus of comet 67P/Churyumov-Gerasimenko from OSIRIS observations. Astron. Astrophys. 583, A32 (2015).

16. Jutzi, M. \& Benz, W. Formation of bi-lobed shapes by sub-catastrophic collisions. A late origin of comet 67P's structure. Astron. Astrophys. 597, A62 (2017).

17. Collins, G. S. Numerical simulations of impact crater formation with dilatancy. J. Geophys. Res. Planets 119, 2600-2619 (2014).

18. Jutzi, M. \& Asphaug, E. The shape and structure of cometary nuclei as a result of low-velocity accretion. Science 348, 1355-1358 (2015).
19. Davidsson, B. J. R. et al. The primordial nucleus of comet $67 \mathrm{P} /$ ChuryumovGerasimenko. Astron. Astrophys. 592, A63 (2016).

20. Morbidelli, A. \& Rickman, H. Comets as collisional fragments of a primordial planetesimal disk. Astron. Astrophys. 583, A43 (2015).

21. Singer, K. N. et al. Impact craters on Pluto and Charon indicate a deficit of small Kuiper belt objects. Am. Astron. Soc. Div. Planetary Sci. Conf. 48 $213.12(2016)$

22. El-Maarry, M. R. et al. Regional surface morphology of comet 67P/ Churyumov-Gerasimenko from Rosetta/OSIRIS images. Astron. Astrophys. 583, A26 (2015).

23. Vincent, J.-B. et al. Large heterogeneities in comet $67 \mathrm{P}$ as revealed by active pits from sinkhole collapse. Nature 523, 63-66 (2015).

24. Libourel, G. et al. Search for primitive matter in the Solar System. Icarus 282, 375-379 (2017).

25. Michel, P., Benz, W., Tanga, P. \& Richardson, D. C. Collisions and gravitational reaccumulation: forming asteroid families and satellites. Science 294, 1696-1700 (2001).

26. Richardson, D. C., Michel, P., Walsh, K. J. \& Flynn, K. W. Numerical simulations of asteroids modelled as gravitational aggregates with cohesion. Planet. Space Sci. 57, 183-192 (2009).

27. Jutzi, M. SPH calculations of asteroid disruptions: the role of pressure dependent failure models. Planet. Space Sci. 107, 3-9 (2015).

28. Cundall, P. A. \& Strack, O. D. L. A discrete numerical model for granular assemblies. Geotechnique 29, 47-65 (1979).

29. Rickman, H. The nucleus of comet Halley: surface structure, mean density, gas and dust production. Adv. Space Res. 9, 59-71 (1989).

30. A'Hearn, M. F. et al. Deep impact: excavating comet Tempel 1. Science 310, 258-264 (2005).

31. A'Hearn, M. F. et al. EPOXI at comet Hartley 2. Science 332, 1396-1400 (2011).

32. Duxbury, T. C., Newburn, R. L. \& Brownlee, D. E. Comet 81P/Wild 2 size, shape, and orientation. J. Geophys. Res. 109, E12S02 (2004).

33. Lamy, P. L., Toth, I. \& Weaver, H. A. Hubble Space Telescope observations of the nucleus and inner coma of comet 19P/1904 Y2 (Borrelly). Astron. Astrophys. 337, 945-954 (1998).

34. Soderblom, L. A. et al. Observations of comet 19P/Borrelly by the Miniature Integrated Camera and Spectrometer aboard Deep Space 1. Science 296, 1087-1091 (2002)

\section{Acknowledgements}

S.R.S. and P.M. acknowledge support from the Centre National d'Études Spatiales, as well as the Academies of Excellence on Complex Systems and Space, Environment, Risk and Resilience of the Initiative d'EXcellence 'Joint, Excellent, and Dynamic Initiative' (IDEX JEDI) of the Université Côte d'Azur. M.J. acknowledges support from the Swiss National Centre of Competence in Research PlanetS, and S.M. acknowledges support from the Jet Propulsion Laboratory. Computation was performed using the YORP computing cluster run by the Center for Theory and Computation at the University of Maryland's Department of Astronomy and the Mésocentre de Calcul Intensif 'Simulations Intensives en Géophysique, Astronomie, Mécanique, et Mathématiques' hosted by the Côte d'Azur Observatory in Nice. For data visualization, the authors made use of the freeware, multiplatform, ray-tracing package, Persistence of Vision Raytracer.

\section{Author contributions}

S.R.S. performed the soft-sphere/ $N$-body numerical simulations, analysed the numerical results and led the research. M.J. performed the SPH numerical simulations and contributed to the analyses. P.M. initiated the collaboration between the institutions to investigate catastrophic impacts as an origin story, and helped steer the study to address questions in the context of Solar System origins. S.M. provided essential and detailed context for the Rosetta findings and outstanding questions regarding comet 67P/G-C. Y.Z. analysed the friction angles and implemented the rolling friction subroutine used D.C.R. guided the development of all $\mathrm{N}$-body-related code. All authors contributed to interpretation of the results and preparation of the manuscript.

\section{Competing interests}

The authors declare no competing interests.

\section{Additional information}

Supplementary information is available for this paper at https://doi.org/10.1038/ s41550-018-0395-2.

Reprints and permissions information is available at www.nature.com/reprints.

Correspondence and requests for materials should be addressed to S.R.S.

Publisher's note: Springer Nature remains neutral with regard to jurisdictional claims in published maps and institutional affiliations. 